\title{
ANÁLISE GEOESTATISTICA APLICADA NA DISTRIBUIÇÃO DE ARBOVIROSES EMERGENTES NO MUNICÍPIO DE CAICÓ - RN
}

\author{
Diógenes Felix da Silva Costa ${ }^{(a)}$; Paulo Jerônimo Lucena Oliveira ${ }^{(b)}$, Diego Emanoel Moreira da \\ Silva $^{(\mathrm{c})}$, Jânio Carlos Fernandes Guedes ${ }^{(\mathrm{d})}$ \\ (a) Coordenador LAMMA/DGC/CERES/UFRN, diogenesgeo@gmail.com \\ (b) LAMMA/DGC/CERES/UFRN, paulojeronimo.geo@gmail.com \\ (c) LAMMA/DGC/CERES/UFRN, diegoemanoel22@gmail.com \\ (d) LAMMA/DGC/CERES/UFRN, janiocf.guedes@ gmail.com
}

\section{Eixo: GEOTECNOLOGIAS E MODELAGENS ESPACIAL EM GEOGRAFIA FÍSICA}

\begin{abstract}
Resumo
A dengue, chicungunya e a zika são uma arbovirose causadas pelo mosquito Aedes aegypti que vem preocupando as autoridades sanitárias de todo o mundo. No Brasil, o vetor encontrou condições favoráveis para sua dispersão em 1976. Frente a isso, o objetivo do trabalho é identificar e demarcar as áreas de ocorrências de doenças decorrentes do mosquito Aedes aegypti no período de 2016, em Caicó. Para tanto, foram utilizados dados referentes às ocorrências de doenças decorrentes do mosquito no município. Os mesmos foram tabulados em planilha do Excel e trabalhadas em ambiente de SIG com o auxilio do software ArcGis 10.3, onde foram especializadas através de técnicas de geoestatística. Como resultados, observou-se que a zonas Oeste e Norte do município apresentaram as maiores ocorrências de doenças. Assim, o uso das geotecnologias mostraram-se eficientes na distribuição e espacialização dos casos podendo servir como estratégia para controle da mesma.
\end{abstract}

Palavras chave: : Geografia da saúde, Arborivoses, Krigagem, SIG.

\section{Introdução}

A dengue é uma arbovirose que vem preocupando as autoridades sanitárias de todo o mundo em virtude de sua circulação nos cinco continentes e do grande potencial para o desenvolvimento de formas graves e letais de doença (FLAUZINO et al., 2009). A doença tornou-se endêmica em 112 países da África, das Américas, da Região Leste do Mediterrâneo, do Sudeste Asiático e do Pacífico Ocidental (SINGHI et al.., 2007) único vetor reconhecido como transmissor do vírus da dengue em nosso meio (CÂMARA et al., 2007). No Brasil, o vetor (Aedes aegypti) encontrou desde sua reintrodução no território nacional, em 1976, condições favoráveis a sua dispersão, como o acelerado processo de urbanização e de crescimento populacionais aliados a ineficiencia dos programas de combate ao vetor (TEIXEIRA et al., 2005; TAULI, 2001). Em outras palavras, no Brasil, o acentuado número de casos de dengue tem sido relacionado, principalmente a fatores que incluem o processo de urbanização desordenada, presença de lixo em quintais e terrenos baldios e variações de temperatura e pluviosidade (CHIARAVALLOTI NETO, 1997; TAUIL,op. cit.). 


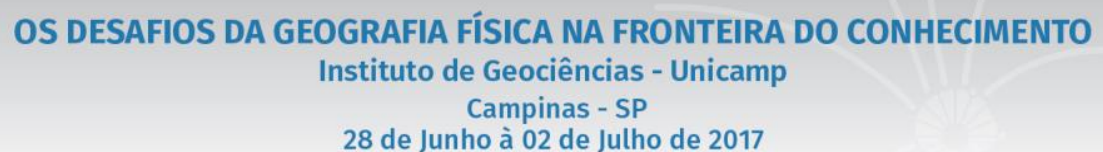

A Organização Mundial de Saúde (1999) estima que cerca de 2,5 bilhões de pessoas que vivem em áreas tropicais e subtropicais estão em estado de risco, e que ocorram anualmente 50 milhões de casos, com cerca de 550 mil hospitalizados e pelo menos 20 mil mortes, tornado-se a doença mais importante no campo das doenças infecciosas.

Na última década, o número de casos novos da dengue diagnosticados no Estado do Rio Grande do Norte, por sua vez, apresentou um acentuado crescimento. Em 2016, foram registrados 1.438 .624 casos prováveis de dengue no país até a Semana Epidemiológica (SE) 37 (03/01/2016 a 17/09/2016), sendo estes todos os casos notificados, exceto os já descartados. O Rio Grande do Norte, por sua vez, está à frente dos demais Estados do Nordeste do Brasil quando o assunto é infestação do mosquito da dengue. Segundo a Secretaria de Estado da Saúde Pública (SESAP), por meio do Programa Estadual de Controle da Dengue (PECD), de acordo com o Boletim Epidemiológico, relativo à semana epidemiológica 37 de 2016, foi notificada até o momento no RN 57.214 casos. Em 2015, no mesmo período, foram registrados 21.513 casos de dengue, ou seja, houve um aumento de $165 \%$ dos casos em relação com o mesmo período analisado. O cenário epidemiológico da dengue no Rio Grande do Norte revela a ocorrência de grande número de óbitos, caracterizando uma situação preocupante para a vigilância epidemiológica uma vez que o óbito por dengue é, em sua maioria, evitável. No ano de 2015 foram notificados 12 óbitos e em 2016 são 57 óbitos, destes, um confirmado para dengue grave e dois descartados.

Dando ênfase ao município de Caicó (área do estudo) pertencente ao estado do Rio Grande do Norte, sendo a principal cidade da microrregião do Seridó Ocidental, na porção centro-sul do estado, distante 269 km da capital do estado, Natal (IBGE, 2010). Torna-se necessário a implantação de medidas de monitoramento e o desenvolvimento de estratégias de controle de focos de proliferação do mosquito vetor, pois no ano de 2016 foram notificados 3.189 casos de Dengue, até a primeira semana do mês de outubro do presente ano citado (SESAP, 2016). Mais recentemente, as geotecnologias vêm sendo utilizadas para auxiliar no mapeamento das ocorrências de casos de doenças e na caracterização epidemiológica de endemias (MEDEIROS-SILVA et al., 2015).

As geotecnologias, por sua vez, também conhecidas como "geoprocessamento", são o conjunto de tecnologias para coleta, processamento, análise e oferta de informações com referência geográfica (ROSA, 2005). Dentre as ferramentas geotecnológicas, os Sistemas de Informações Geográficas (SIG) podem auxiliar no monitoramento da doença e no fornecimento de informações atualizadas, indicando a real situação epidemiológica numa determinada localidade (BARCELLOS, 2005).

Logo, a distribuição espacial das doenças pode ser mapeada e analisada usando o SIG, capaz de armazenar informações geográficas, correlacioná-las com dados tabulares, podendo ser usado para coleta, 
armazenagem, interrogação e exibição dos dados espaciais, ajudando a determinar a localização espacial das doenças e a análise gráfica dos indicadores epidemiológicos (BESSA-JUNIOR et al., 2013).

Segundo a Organização Mundial de Saúde (OMS), trata-se de uma ferramenta eficaz na gerência do Programa Nacional de Controle da Dengue (PNCD). Face ao exposto, o conhecimento da relação espacial dos casos de dengue pode propiciar maior entendimento sobre a dinâmica de transmissão e seu controle (RIBEIRO et al., 2006). Nessa perspectiva, o uso das geotecnologias se tornou fundamental para aplicação na área de saúde, com destaque para análises da distribuição espacial de endemias (SILVA et al., 2012).

Diante do que foi exposto, o objetivo do trabalho é identificar e demarcar as áreas de ocorrências de casos de dengue, chicungunya e zika, diagnosticados no período de 2016, na zona urbana do município de Caicó.a 5 páginas para resumos expandidos, incluindo tabelas, figuras e referências bibliográficas. As seções deverão ser numeradas sequencialmente. Os agradecimentos e as entidades financiadoras deverão ser incluídos no final do texto.

\section{Materiais e Métodos}

\section{1 Área de estudo}

A área de estudo compreende a zona urbana do município de Caicó localizado na Microrregião do Seridó Ocidental do Estado do Rio Grande do Norte (6 $6^{\circ} 27^{\prime} \mathrm{S}$ e $\left.37^{\circ} 5^{\prime} \mathrm{O}\right)$, o mesmo apresenta área territorial em torno de $1.228 \mathrm{~km}^{2}$, em uma população média estimada em 67.000 habitantes, uma densidade media de aproximadamente 51 (hab./ $\mathrm{km}^{2}$ ) (IBGE, 2016). O Índice de Desenvolvimento Humano Municipal (IDHM) de Caicó é de 0,710, considerado "alto" pelo Programa das Nações Unidas para o Desenvolvimento (PNUD) sendo o quarto maior do estado do Rio Grande do Norte (PNUD, 2016) (Figura 01).

A escolha da área de estudo se deu pelo fato do município de Caicó está enquadrado, assim como outros 42 municípios do RN, como vulnerável para ocorrência de epidemia de dengue no estado (Sesap, 2014). 

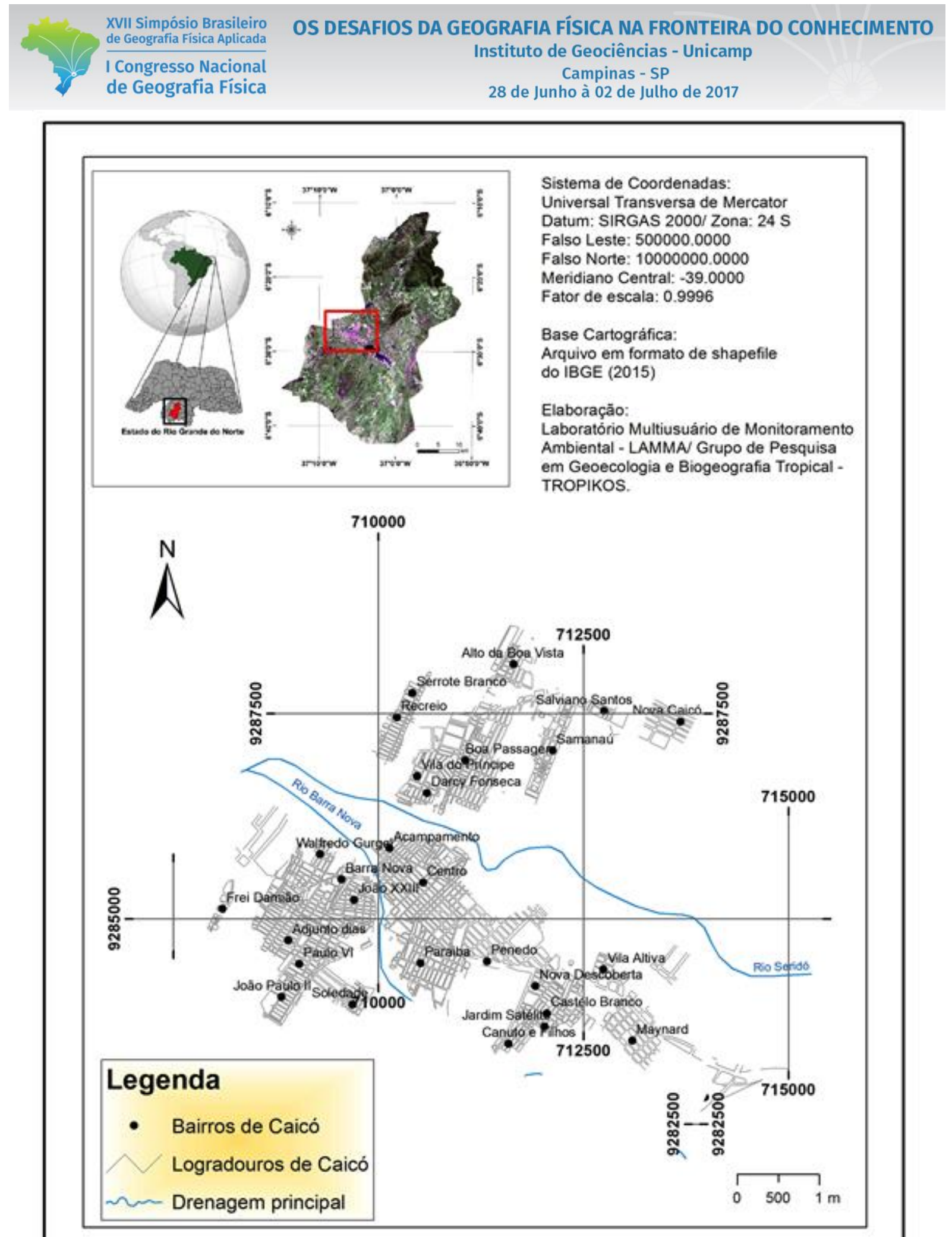

Figura 01 - Mapa de localização da zona urbana do municipio de Caicó/RN 


\subsection{Procedimentos Metodológicos}

Primeiramente realizou-se uma revisão bibliográfica e cartográfica, com vistas à obtenção de aporte teórico sobre a temática desenvolvida e sobre os procedimentos adotados dentro da escala da pesquisa.

Trata-se de um estudo descritivo, realizado na área urbana do município de Caicó - RN, dessa forma, foram utilizados arquivos vetorias referentes aos logradouros do municipio disponivel no sítio do IBGE (Instituto Brasileiro de Geografia e Estatística) como também de dados referentes ao número de imóveis, inspecionados e imóveis com focos da dengue e Zica vírus no município de Caicó - RN, disponível na Secretaria Municipal de Saúde do Município). Os dados foram tabulados e armazenados no software Excel 2010 contendo o nome dos bairros, sua localização, número de imóveis total e número de imóveis inspecionados juntamente com o número de imóveis com ocorrências da dengue e sua porcentagem.

Posteriormente, esses dados foram exportados para um ambiente de SIG, nos quais foram georreferenciadas na grade de coordenadas UTM (Universal Transversa de Mercartor), a partir do Datum SIRGAS 2000, Zona 24S, onde, gerou-se um arquivo em formato de vetor (shapefile de pontos) no software Arcgis 10.3 (versão acadêmica).

Logo após, gerou-se um mapa de zonas de maior incidência de focos de dengue na zona urbana do município de Caicó - RN. Por fim gerou-se também um mapa com a espacialização dos focos de dengue e zika na zona urbana do município a partir da interpolação dos dados através de técnicas de Krigagem dos dados.

A análise espacial tem como ênfase mensurar propriedades e relacionamentos, levando-se em consideração a localização geográfica do fenômeno em estudo de forma explícita. A análise espacial pode ser definida como uma técnica que busca descrever os padrões existentes nos dados espaciais e estabelecer, preferencialmente de forma quantitativa, os relacionamentos entre as diferentes variáveis geográficas (MEDEIROS, op. Cit.).

\section{Resultados}

A partir dos dados coletados na secretaria municipal de sáude de Caicó, verificou-se a existência de focos de dengue em 28 bairros da zona urbana do município, distribuidos na zona central e nas zonas limítrofes (Norte, Sul, Leste e Oeste). Pode-se constatar, uma grande variação em relação ao total de imóveis como o foco da doença por bairro, desde o valor mínimo de 1 imovél no bairro Serrote Branco, até o índece mais elevado no bairro Walfredo Gurgel, com o total de 310 imovéis com o foco da doença (Figura 02). 

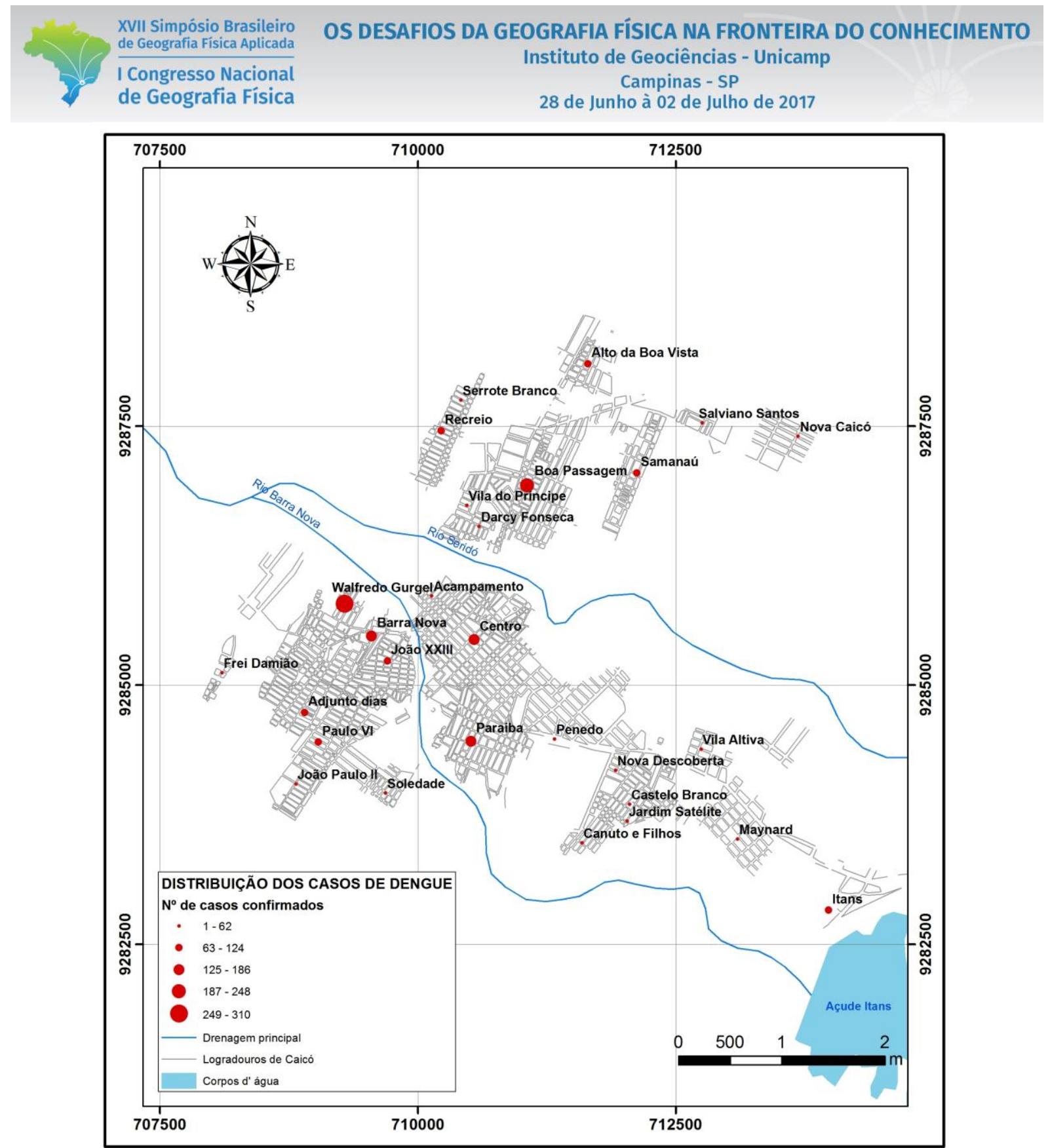

Figura 02 - Mapa de distribuição quantitativa de focos de dengue na zona urbana do município de Caicó - RN, no ano de 2016.

Desde modo, de acordo com os dados analisados pode-se dividir o resultado de imóveis por cada porção da zona urbana do município, a primeira zona é a Central com 02 bairros (Centro e Acampamento), a zona Norte abrangendo 09 bairros (Alto da Boa Vista, Boa Passagem, Darcy Fonseca, Nova Caicó, Recreio, Salviano Santos, Samanaú, Serrote Branco e Vila do Príncipe), a zona Sul com 02 bairros (Paraíba e Soledade), a zona Leste, preenchida com 08 bairros (Canutos e Filhos, Castelo Branco, Itans, Jardim Satélite, Maynard, Nova Descoberta, Penedo e Vila Altiva) e a zona Oeste, compreendendo 07 bairros (Adjunto Dias, Barra Nova, Frei Damião, João Paulo II, João XXIII, Paulo VI e Walfredo Gurgel). 
Figura 03 - Distribuição dos casos de dengue no município de Caicó-RN, nas zonas Leste (A) e Oeste (B), no ano de 2016.

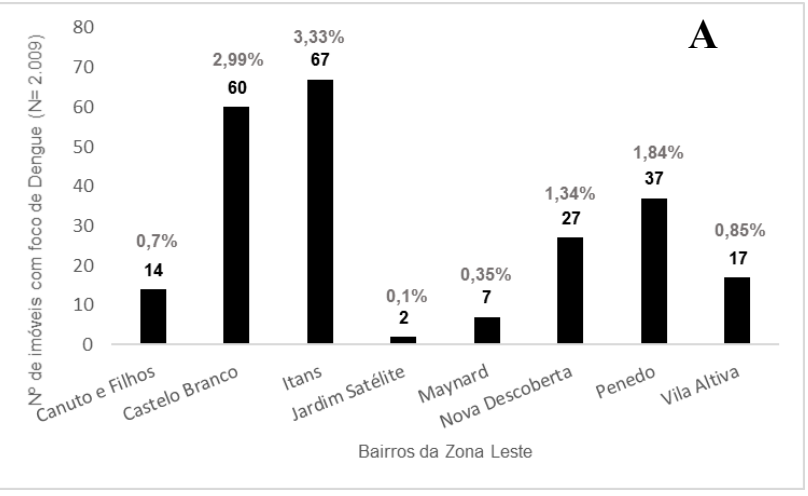

$\mathbf{C}_{1 . .}$

Pode-se verificar, que nas duas zonas houve

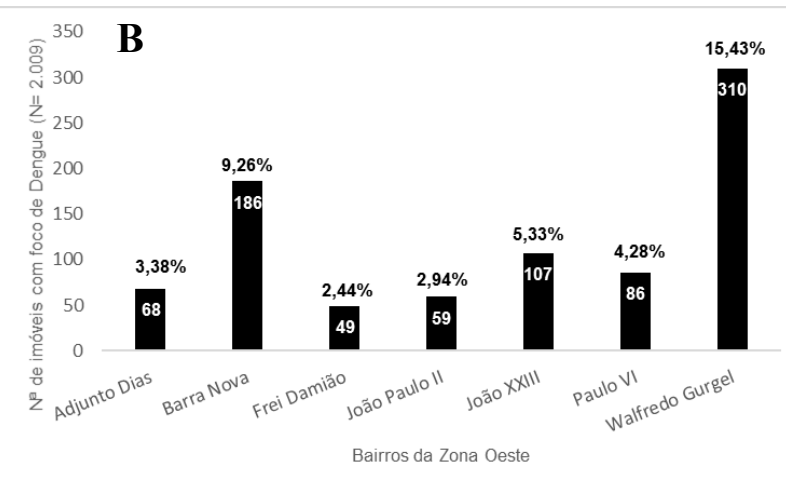

D variação em relação aos casos de uma

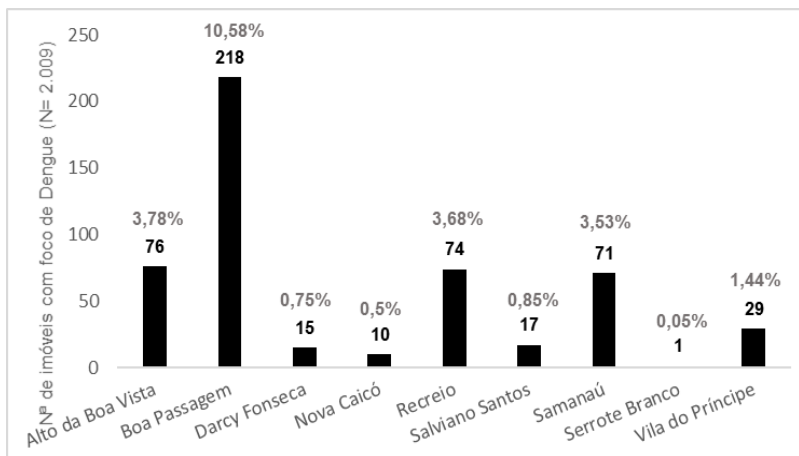

Bairros da Zona Norte

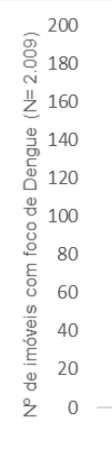

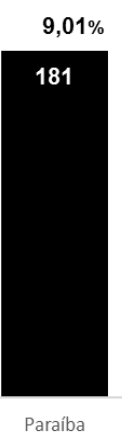

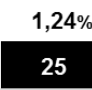

Soledade

focos de dengue identificados, na zona Leste os bairros Itans e Castelo Branco, sequencialmente com 67 $(3,33 \%)$ e $60(2,99 \%)$ casos foram os setores com maior ocorrência de casos de dengue, ainda no campo Leste o destaque positivo fica pelo bairro Jardim Satélite com a ocorrência de apenas 2 focos. Em relação a zona Oeste, esse é o setor com maior índice de focos de dengue, com um total de 865 focos de dengue distribuídos em 7 bairros, logo o destaque com maior ocorrência de dengue fica pelo bairro Walfredo Gurgel com 310 casos confirmados, cerce de 15,43\% de todos os casos da cidade, sendo este o bairro com maior presença de focos em toda a zona urbana do município.

Além disso, pode-se também analisar através do referido estudo, qual zona do município está mais infectada pelos focos dos mosquitos da dengue. O setor que registrou mais indícios de casos de dengue foi a zona Oeste com cerca de $43,06 \%$ de todos os casos urbanos do município, seguido da zona Norte com $25,43 \%$ de focos de casos de dengue, já as zonas Sul e Leste obtiveram um resultado semelhante em relação ao número de focos, $10,65 \%$ e $10,25 \%$ respectivamente do total, e em último lugar se encontra a 
porção central da cidade com 9,76\% de todos os casos identificados na porção urbana de Caicó (Figura 04).

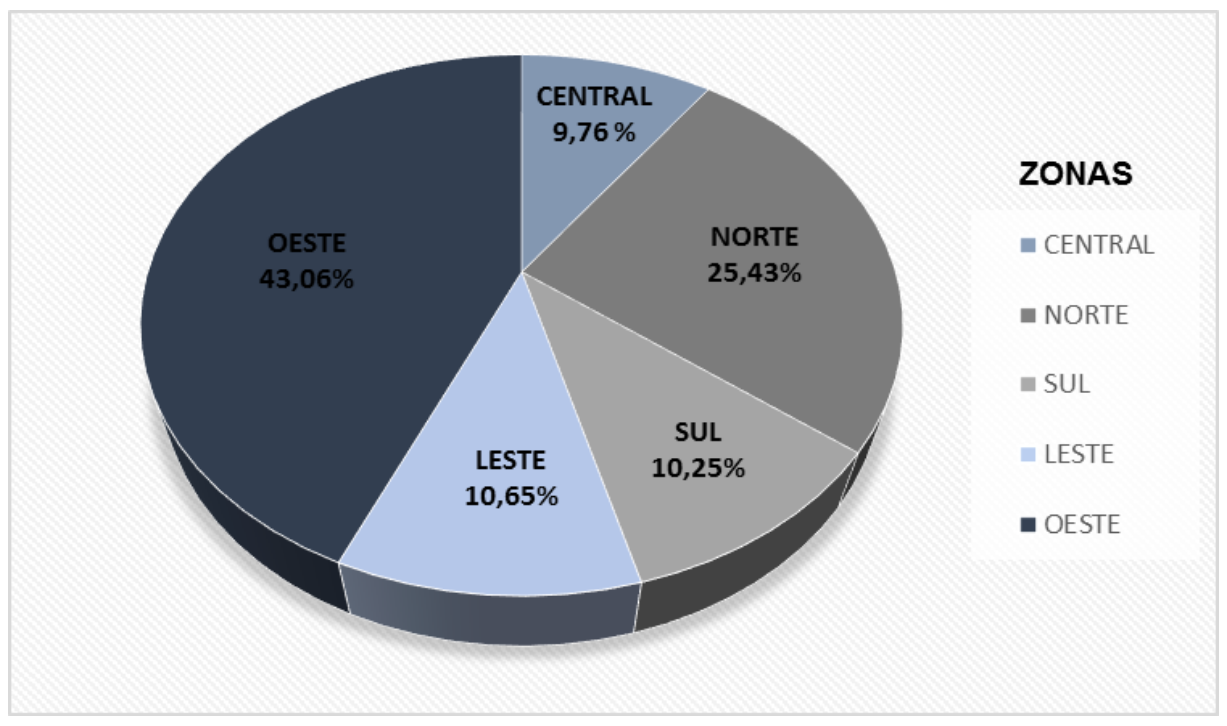

Figura 04 - Distribuição os percentuais dos casos de dengue no município de Caicó - RN, por zonas, no ano de 2016. A partir dos dados, gerou-se também um mapa com a espacialização dos focos de dengue no município a partir da Krigagem dos dados, onde se percebeu a maior concentração de casos na região centro oeste da zona urbana do município, como expressa os resultados gráficos anteriores e confirmado na interpretação gráfica seguinte (Figura 05).

Na sequencia foi análisada a segunda arbovirose, o zika vírus, variavelmente os dados do zika vírus foram realizados nas UBS (Unidade Básica de Saúde) e não em visitas residenciais como no caso da dengue. Logo, foram identificados casos de zika vírus em pessoas de 12 bairros da cidade computando um total de teste positivo da arboviroses em 34 casos distribuídos em todas as zonas do município (Tabela I).

Tabela I: Distribuição dos casos de zika no município de Caicó-RN, por zona e bairros, no ano de 2016. 


\begin{tabular}{|c|c|c|c|c|c|}
\hline \multirow{2}{*}{\multicolumn{2}{|c|}{$\begin{array}{l}\text { XVII Simpósio Brasileiro } \\
\text { de Geografia Fisica Aplicada } \\
\text { I Congresso Nacional } \\
\text { de Geografia Física }\end{array}$}} & \multirow{2}{*}{\multicolumn{4}{|c|}{$\begin{array}{l}\text { OS DESAFIOS DA GEOGRAFIA FÍSICA NA FRONTEIRA DO CONHECIMENTO } \\
\text { Instituto de Geociências - Unicamp } \\
\text { Campinas - SP } \\
28 \text { de Junho à } 02 \text { de Julho de } 2017\end{array}$}} \\
\hline & & & & & \\
\hline \multirow{2}{*}{ Zona } & \multicolumn{2}{|c|}{ Coordenadas Projetadas } & \multirow{2}{*}{ Bairros } & \multirow{2}{*}{ Casos } & \multirow{2}{*}{$\begin{array}{c}\text { Valores } \\
(\%)\end{array}$} \\
\hline & $\mathbf{X}$ & $\mathbf{Y}$ & & & \\
\hline \multirow{2}{*}{ CENTRAL } & 710545 & 9285439 & Centro & 3 & 8,82 \\
\hline & 710134 & 9285859 & Acampamento & 4 & 11,76 \\
\hline \multirow{2}{*}{ NORTE } & 713686 & 9287400 & Nova Caicó & 3 & 8,82 \\
\hline & 710227 & 9287452 & Recreio & 1 & 2,94 \\
\hline \multirow{2}{*}{ SUL } & 710516 & 9284459 & Paraíba & 6 & 17,65 \\
\hline & 709686 & 9283957 & Soledade & 3 & 8,82 \\
\hline \multirow{3}{*}{ LESTE } & 713673 & 9283069 & Canutos e Filhos & 1 & 2,94 \\
\hline & 713984 & 9282825 & Itans & 1 & 2,94 \\
\hline & 711916 & 9284179 & Nova Descoberta & 1 & 2,94 \\
\hline \multirow{3}{*}{ OESTE } & 709552 & 9285476 & Barra Nova & 1 & 2,94 \\
\hline & 709036 & 9284448 & Paulo VI & 5 & 14,71 \\
\hline & 709289 & 9285782 & Walfredo Gurgel & 5 & 14,71 \\
\hline TOTAL & - & - & - & 34 & 100,00 \\
\hline
\end{tabular}

Através da quantificação dos dados da arboviroses zika, tem-se na região Central da cidade, a ocorrência de 07 casos identificados, com 03 (8,82\%) no Centro da cidade e 04 (11,76\%) no bairro do Acampamento. A zona Norte obteve 04 casos de zika vírus, 03 (8,82\%) na Nova Caicó e 01 (2,94\%) no bairro do Recreio, já em relação a zona Sul, foi a segunda porção da cidade com mais casos identificados, onde entre os dois bairros dessa região, encontra-se o bairro Paraíba com o bairro que mais casos foram identificados 06, tendo $17,65 \%$ do total de todos os casos do município, o outro bairro foi a soledade com $03(8,82 \%)$ casos. Os últimos dados coletados foram da arbovirose Chikugunya, onde semelhantemente aos casos de Dengue, registraram-se casos da doença em todos os bairros da zona urbana do município. Sendo um total de 1.070 casos de Chikugunya em todas as regiões da cidade. Logo, a zona Central urbana do município, é representada pelos bairros Centro, onde foram registrados 120 (11,21\%) casos, sendo esse o quinto bairro com maior número de ocorrências na cidade, e o Acampamento com 15 casos, com aproximadamente $1 \%$ de todos os casos, mais precisamente $1,40 \%$ das ocorrências (Tabela II).

Figura 05: Mapa com a espacialização dos casos de dengue no município de Caicó - RN. 

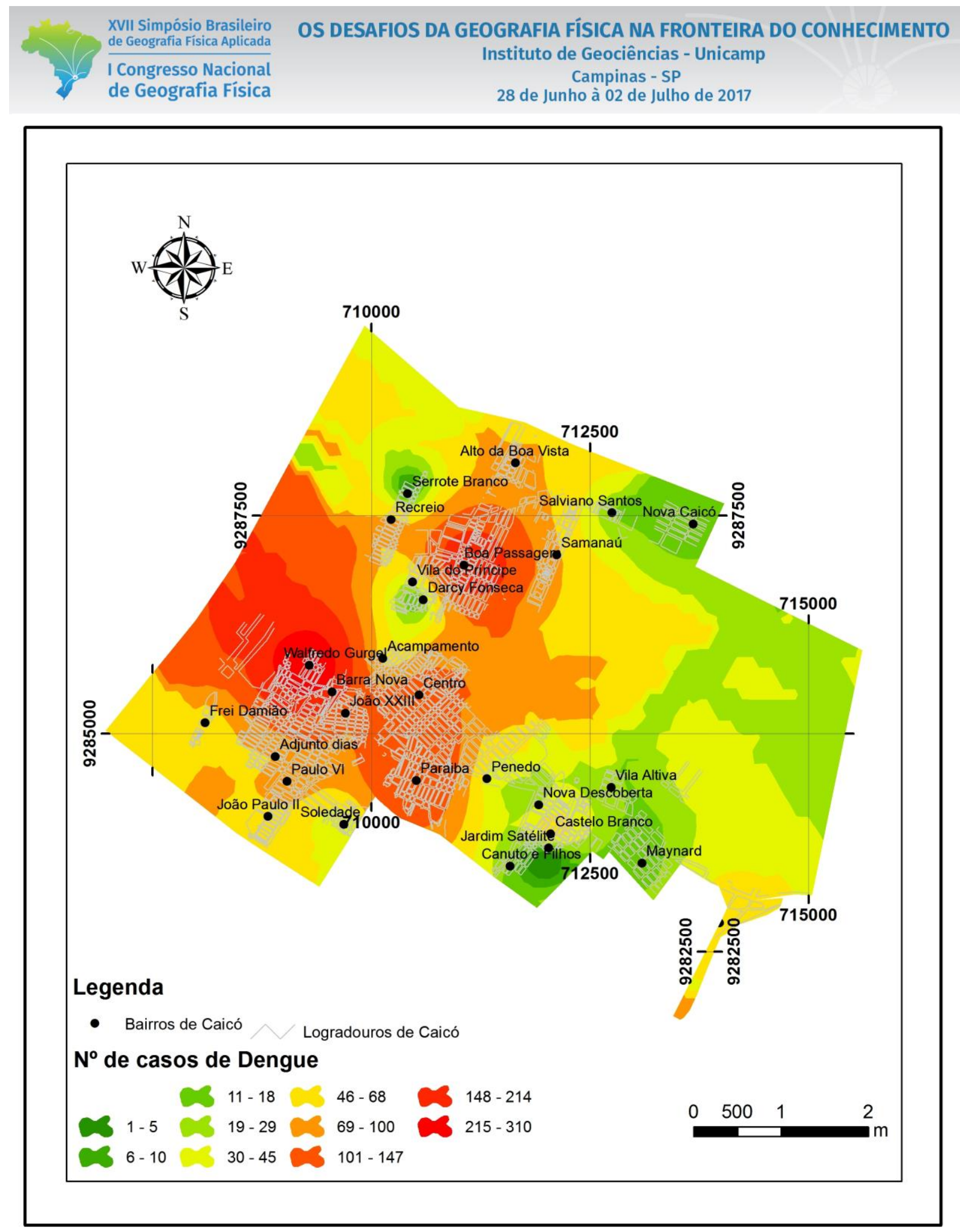

Tabela II - Distribuição dos casos de Chikugunya no município de Caicó-RN, por zona e bairros, no ano de 2016. Fonte: Elaborado através dos dados da Secretaria Municipal de Saúde - Caicó - RN, do ano 2016. 
XVII Simpósio Brasileiro de Geografia Física Aplicada

I Congresso Nacional de Geografia Física
OS DESAFIOS DA GEOGRAFIA FÍSICA NA FRONTEIRA DO CONHECIMENTO

Instituto de Geociências - Unicamp

Campinas - SP

28 de Junho à 02 de Julho de 2017

\begin{tabular}{|c|c|c|c|c|c|}
\hline \multirow{2}{*}{ Zona } & \multicolumn{2}{|c|}{ Coordenadas Projetadas } & \multirow{2}{*}{ Bairros } & \multirow{2}{*}{ Casos } & \multirow{2}{*}{$\begin{array}{c}\text { Valores } \\
(\%)\end{array}$} \\
\hline & $\mathrm{X}$ & $\mathbf{Y}$ & & & \\
\hline \multirow{2}{*}{ CENTRAL } & 710545 & 9285439 & Centro & 120 & 11,21 \\
\hline & 710134 & 9285859 & Acampamento & 15 & 1,40 \\
\hline \multirow{9}{*}{$\begin{array}{l}\text { w } \\
\frac{\mathbf{x}}{\mathbf{g}} \\
\mathbf{z}\end{array}$} & 711650 & 9288100 & Alto da Boa Vista & 88 & 8,22 \\
\hline & 711061 & 9286928 & Boa Passagem & 104 & 9,72 \\
\hline & 710595 & 9286531 & Darcy Fonseca & 1 & 0,09 \\
\hline & 713686 & 9287400 & Nova Caicó & 25 & 2,34 \\
\hline & 710227 & 9287452 & Recreio & 6 & 0,56 \\
\hline & 711924 & 9286608 & Salviano Santos & 4 & 0,37 \\
\hline & 712124 & 9287047 & Samanaú & 14 & 1,31 \\
\hline & 710416 & 9287749 & Serrote Branco & 1 & 0,09 \\
\hline & 710474 & 9286735 & Vila do Príncipe & 7 & 0,65 \\
\hline \multirow{2}{*}{ क } & 710516 & 9284459 & Paraíba & 141 & 13,18 \\
\hline & 709686 & 9283957 & Soledade & 12 & 1,12 \\
\hline \multirow{8}{*}{ 惢 } & 713673 & 9283069 & Canuto e Filhos & 8 & 0,75 \\
\hline & 712054 & 9283848 & Castelo Branco & 4 & 0,37 \\
\hline & 713984 & 9282825 & Itans & 2 & 0,19 \\
\hline & 712029 & 9283685 & Jardim Satélite & 3 & 0,28 \\
\hline & 713098 & 9283513 & Maynard & 7 & 0,65 \\
\hline & 711916 & 9284179 & Nova Descoberta & 11 & 1,03 \\
\hline & 711326 & 9284479 & Penedo & 6 & 0,56 \\
\hline & 712746 & 9284381 & Vila Altiva & 32 & 2,99 \\
\hline \multirow{7}{*}{ 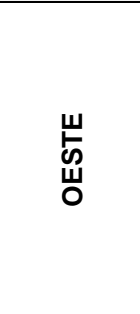 } & 708903 & 9284734 & Adjunto Dias & 15 & 1,40 \\
\hline & 709552 & 9285476 & Barra Nova & 125 & 11,68 \\
\hline & 708100 & 9285119 & Frei Damião & 12 & 1,12 \\
\hline & 709415 & 9284955 & João Paulo II & 19 & 1,78 \\
\hline & 709706 & 9285231 & João XXIII & 29 & 2,71 \\
\hline & 709036 & 9284448 & Paulo VI & 125 & 11,68 \\
\hline & 709289 & 9285782 & Walfredo Gurgel & 134 & 12,52 \\
\hline TOTAL & - & - & - & 1.070 & 100 \\
\hline
\end{tabular}

\section{CONCLUSÕES}

O estudo da distribuição espacial da dengue no município de Caicó utilizando o SIG, mostrou-se passível de uso e um instrumento útil na vigilância epidemiológica, onde a partir das ferramentas de distribuição quantitativa por pontos e da Krigagem, observou-se que, através dos cartogramas se pode analisar os dados tantos de forma quantitativa em forma de distribuição de ponto, quanto pela tonalidade de cores fortes (vermelho) e fracas (verde), onde de acordo com a tonalidade se interpreta a maior ocorrência de casos. 


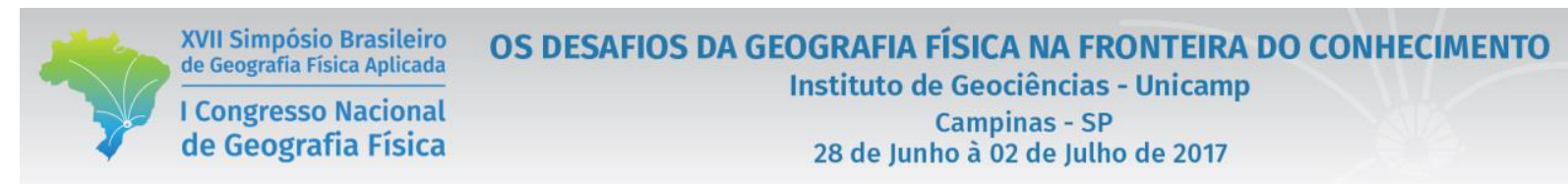

Pode-se constatar através das análises gráficas e cartográficas, que os maiores casos de focos de doenças decorrentes no mosquito Aedes Aegypti na cidade esta entrelaçadas entre a porção Oeste e Norte, mais com um maior destaque na zona Oeste da cidade. Ainda em relação a parte gráfica pode-se ter uma noção mais aprimorada de qual zona da cidade ocorre os maiores casos, como já mencionado na porção Oeste.

Portanto, frisa-se que é necessário centrar e facilitar as ações com a Vigilância Sanitária de Caicó, principalmente nos bairros da zona Oeste que abrange uma população de caráter ribeirinhas nas margens dos Rios Seridó e Barra Nova, que são carentes de pavimentação e saneamento, além de nessas áreas se localiza distribuição irregular de lixo (famosos lixões). Assim a busca por estratégias de combate junto às áreas prioritárias, através de políticas públicas e programas por órgãos públicos.

\section{Bibliografia}

Atlas do Desenvolvimento Humano. Programa das Nações Unidas para o Desenvolvimento (PNUD). Perfil do IDH-M caicoense 2016. Disponível em: <http://www.atlasbrasil.org.br/2013/pt/perfil_m/caico_rn> Acesso em 18 de novembro de 2016.

BARCELLOS, C. et al. Identificação de locais com potencial de transmissão de dengue em Porto Alegre através de técnicas de geoprocessamento. Revista da Sociedade Brasileira de Medicina Tropical, [S.1.], v. 38, n. 30, p. 50-246, 2005.

BESSA-JÚNIOR, F. N; NUNES, R. F. F; SOUZA, M. A; MEDEIROS, A. C; MARINHO, M. J. M; PEREIRA, W. O. Distribuição espacial da dengue no município de Mossoró, Rio Grande do Norte, utilizando o Sistema de Informação Geográfica. Rev Bras Epidemiol. V.16, n.3: p.603-10, 2013.

CAMARA, F. P. et al. Estudo retrospectivo (histórico) da dengue no Brasil: características regionais e dinâmicas. Revista da Sociedade Brasileira de Medicina Tropical, 40(2), 192- 196, 2007.DIAGNÓSTICO do setor editorial brasileiro. São Paulo: Câmara Brasileira do Livro, 1993. 64 p. PERFIL da administração pública paulista. 6. ed. São Paulo: Fundap, 1994. 317 p.

CHIARAVAlloti NeTO, F. Descrição da colonização de Aedes aegypti na região de São José do Rio Preto, São Paulo. Revista da Sociedade Brasileira de Medicina Tropical, Uberaba, v. 30, n. 4, p. 279-285, 1997.

FLAUZINO, R. F; SOUZA-SANTOS, R; OLIVEIRA, R. M. Dengue, geoprocessamento e indicadores socioeconômicos e ambientais: um estudo de revisão. Revista Panamericana de Salud Pública/Pan Am J Public Health v. 25 n.5, 2009. P. 456-461.

INSTITUTO BRASILEIRO DE GEOGRAFIA E ESTATÍSTICA - IBGE, 2010. Cidades: Caicó, censo $\begin{array}{lcc}\text { demográfico } & \mathbf{2 0 1 0} & \text { Disponível } \\ \text { http://www.cidades.ibge.gov.br/painel/historico.php?lang=\&codmun=240200\&search=rio-grande-do- }\end{array}$ norte|caico|infograficos:-historico>. Acesso em: 19 dez. 2016. 
MEDEIROS-SILVA, A; MARQUES-SILVA, R; ALMEIDA, C. A. P; CHAVES, J. J. S. Modelagem geoestatística dos casos de dengue e da variação termopluviométrica em João Pessoa, Brasil. Revista Soc. \& Nat., Uberlândia, 27 (1): 157-169, jan/abr/2015.

RIBEIRO, A. F; MARQUES, G. R. A. M; VOLTOLINI, J. C; CONDINO, M. L. Associação entre incidência de dengue e variáveis climáticas. Revista Saúde Pública, 40(4), 671-676, 2006.

ROSA, R. Geotecnologias na geografia aplicada. Revista do Departamento de Geografia, v.16: p. 81-90. 2005.

SILVA, R. M; PINTO, H. R. F; COSTA, S. G. F; PINTO, K. R. F. Modelagem geoespacial e temporal da hanseníase entre 2001 e 2011 no município de Bayeux, Paraíba. Hygeia, v.8, n.15, p.89-103, 2012.

SINGHI, S; KISSOON, N; BANSAL, A. Dengue and dengue hemorrhagic fever: management issues in an intensive care unit. Jornal de Pediatria, vol. 83, $\mathrm{n}^{\text {o }}$ 2. p. 22-35, 2007.BRASIL. Constituição (1988). Emenda constitucional n. ${ }^{\circ}$, de 9 de novembro de 1995. Lex: legislação federal e marginália, São Paulo, v. 59, p. 1966, out./dez. 1995.

TAULI, P. L. Urbanização e ecologia do dengue. Cad. Saúde Pública, Rio de Janeiro, ed. 17: p. 99-102, 2001.

TEIXEIRA, M. G; COSTA, M. C. N; BARRETO, M. L; MOTA, E. Dengue and dengue hemorragic fever epidemics in Brasil: what research is needed based on trends, surveillance, and control experiences? Cad. Saúde Pública, Rio de Janeiro, ed. 21, v. 5: 1307-1315, 2005.FAZENDA, I. C. Arantes. Interdisciplinaridade: um projeto em parceria. 6. ed. São Paulo: Loyola, 2007.

WORLD HEALTH ORGANIZATION et al. Strengthening implementation of the global strategy for dengue fever/dengue haemorrhagic fever prevention and control. Report of the Informal Consultation, 18-20 October 1999, WHO HQ, Geneva, Switzerland. Strengthening implementation of the global strategy for dengue fever/dengue haemorrhagic fever prevention and control. Report of the Informal Consultation, 18-20 October 1999, WHO HQ, Geneva, Switzerland., 2000. 